\title{
The Fracture of a Low Carbon Tempered Martensite*
}

\author{
By Tohru Inoue**, Shôichi Matsuda**, \\ Yoshihiro Okamura** and Kôichi Aoki****
}

\begin{abstract}
An investigation was made of the relation between microstructure and fracture characteristics of a low carbon tempered martensite. It was found in a previous paper that the cleavage crack traverses many martensite plates or laths in a linear fashion. On the analogy of the ferritic steel, the effective grain is defined as a region in which cleavage crack runs nearly straight. It is shown that the microstructure corresponding to the effective ferrite grain is the 'co-variant packet' extending throughout a bundle of the martensite. The reason why the structure consisting of fine martensites fractures with a large cleavage facet was found to be due to the fact that most of martensite plates or laths in a packet have the same crystallographic orientation and the cleavage plane $\{100\}_{\alpha}$ is quasi-continuous throughout the packet.
\end{abstract}

(Received September 8, 1969)

\section{Introduction}

The relation between the microstructure and the fracture characteristics of transformation products from austenite is very umbigious; the only clearly known fact is that ferrite grain boundaries act as the resistance to the

\section{Experimental}

$2 \mathrm{mmV}$-notched Charpy test data on a vacuum melted steel were obtained for three austenite grain sizes. The composition of the materials and the treatments used are listed in Tables 1 and $2^{\dagger \dagger}$.

Table 1 Chemical composition of the material used (wt\%)

\begin{tabular}{|c|c|c|c|c|c|c|c|c|c|c|c|}
\hline C & $\mathrm{Mn}$ & $\mathrm{Si}$ & Mo & $\mathrm{Ni}$ & $\mathrm{Cr}$ & $\mathrm{Al}$ & $\mathrm{P}$ & $\mathrm{S}$ & 0 & $\mathbf{N}$ & $\mathbf{B}$ \\
\hline 0.12 & 0.5 & 0.2 & 0.41 & 2.4 & 0.95 & 0.017 & 0.002 & 0.004 & 0.003 & 0.0022 & 0.0003 \\
\hline
\end{tabular}

propagation of a cleavage crack in ferritic steels and therefore the ductile to brittle transition temperature is lower for the steel with small ferrite grains than for that with large ones ${ }^{(1)}$. Martensitic ${ }^{\dagger}$ (or tempered martensitic) and bainitic steel have very complicated microstructures and little has been known about the nucleation site of a crack and the factor controlling the propagation of the cleavage crack.

In a previous paper $^{(2)}$ it was shown that in the lowcarbon tempered martensite, (1) the cleavage microcrack initiates preferentially in the first-formed martensite, (2) the transition temperature $v \operatorname{Tr}_{E}$ is varied with the prior austenite grain size but the size (width) of the first-formed martensite is little affected by the austenite grain size, and (3) good correlation was obtained between the 'mean length of cleavage crack' $l$ (defined as the mean spacing between two neighboring heavy 'tear lines') and the transition temperature $v T_{E}$, suggesting that facet size $\langle l\rangle$ is a dominant factor for cleavage fracture.

The purpose of the present investigation is to indicate how the above-mentioned tear line is directly correlated to the microstructure and to explain this relation in terms of the crystallographic characteristic of the low carbon martensite.

This paper was presented at the Spring Meeting of the Japan Institute of Metals, March, 1969, Tokyo, Japan.

** Tokyo Research Institute, Yawata Iron and Steel Co., Ltd., Ida, Kawasaki, Japan.

*** Tokyo Research Institute, Yawata Iron and Steel Co., Ltd., Ida, Kawasaki. Present address: Yawata Area Works, Yawata Iron and Steel Co., Ltd., Kitakyusyu, Japan.

(1) For example, N. J. Petch : Fracture, John Wiley and Sons, New York, (1959), p. 54.
Table 2 Heat treatment and austenite grain size

\begin{tabular}{|c|c|c|c|c|}
\hline \multirow{2}{*}{ Specimen } & \multicolumn{3}{|c|}{ Heat treatment } & \multirow{2}{*}{$\begin{array}{l}\text { Austenite } \\
\text { grain size } \\
\text { number }\end{array}$} \\
\hline & Austenitizing & Quenching & Tempering & \\
\hline A & $1300^{\circ} \mathrm{C} \times 30 \mathrm{~min}$ & I.B.Q.* & $\begin{array}{l}600^{\circ} \mathrm{C} \\
\quad \times 1 \mathrm{hr}\end{array}$ & ASTM-1 \\
\hline B & $1000^{\circ} \mathrm{C} \times 30 \mathrm{~min}$ & I.B.Q. & $\begin{array}{l}600^{\circ} \mathrm{C} \\
\quad \times 1 \mathrm{hr}\end{array}$ & $" 1$ \\
\hline G & $\begin{array}{c}1000^{\circ} \mathrm{C} \times 30 \mathrm{~min} \\
\text { I.B.Q. } \\
33 \% \text { cold rolled } \\
835^{\circ} \mathrm{C} \times 30 \mathrm{sec}\end{array}$ & I.B.Q. & $\begin{array}{l}600^{\circ} \mathrm{C} \\
\times 1 \mathrm{hr}\end{array}$ & " 12 \\
\hline
\end{tabular}

* Iced brine quenching

Microfractographic, optical and electron-microscopic observations were carried out together with the selected area electron diffraction.

\section{Results}

\section{Heavy tear lines on the fracture surface}

Photo. 1 shows an example how the fracture surface is affected by the prior austenite grain size of the tempered martensite. It is clearly seen that the density of the heavy tear lines decreases as the austenite grain size

$\dagger$ The crystallography and morphology of martensite is not changed by tempering. Therefore, the term 'martensite' will be used hereafter as the abbreviation of 'tempered martensite', because there is no fear of misunderstanding.

(2) S. Matsuda, T.Inoue and M. Ogasawara : Trans. JIM, 9(1968), 343.

it These are of the same material and treatments as used in the previous report ${ }^{(2)}$ 


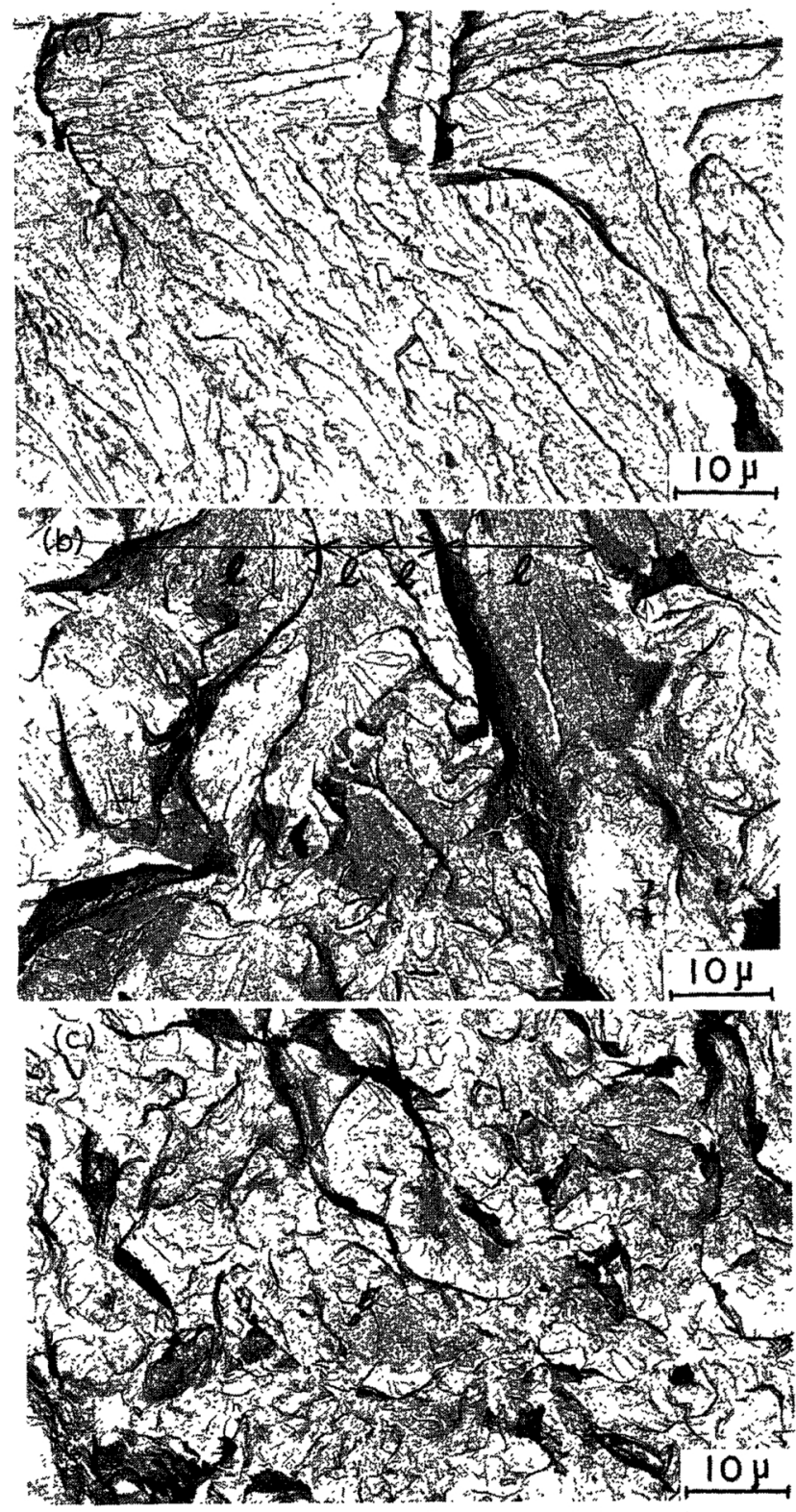

Photo. 1 Fracture surface of the tempered low carbon martensite with various austenite grain sizes (a): Specimen A (b) : Specimen B (c): Specimen C increases, while there are no differences as to many small cleavage steps or tear lines between fine and coarse austenite grains. Spacings between two neighbouring heavy tear lines on the fracture surface were measured and their distributions are illustrated in Fig. 1. Plotting $v \operatorname{Tr}_{E}$ against $\langle l\rangle^{-1 / 2}(\langle l\rangle$ is the statistical means of $l)$, a linear relationship can be obtained as shown in Fig. 2 .

Spacings $l$ were also measured for the untempered martensite, and they are illustrated in Fig. 3. As can be clearly seen from Fig. 1 and Fig. 3, tempering treatment at $600^{\circ} \mathrm{C}$ (for $1 \mathrm{hr}$ ) did not bring about any substantial change in the heavy tear line, suggesting that the characteristic of the heavy tear line originates from the crystallographic characteristic of martensite itself.

\section{Relation between heavy tear line and microstructure}

From the examination of the fracture surface subjected to etching (e.g. Photo. 2), it was found that (1) on the boundary of "bundles" was always observed a heavy tear line (the group of parallel martensite plates or laths is designated "a bundle"). (2) On the prior austenite grain boundary were observed heavy tear lines in most cases. But in some cases where the fracture surfaces were very flat across the austenite grain boundary, no tear lines were observed on the boundary. (3) Within a bundle were also observed heavy tear lines as well as tiny tear lines or cleavage steps along the martensite plates or laths. It seems as if these heavy tear lines were not correlated with any microstructure.

The heavy tear line of type (3) were examined in more detail and found to be correlated with particular microstructures of the low carbon martensite as follows :

Within a bundle of the low carbon martensite there appears a stripe pattern with black and white contrast when the specimen is etched with a special etching reagent ${ }^{\dagger}$. This stripe pattern was confirmed to be due to the crystallographic orientation dependence of etching. Namely the white etched area corresponds to one of two particular crystallographic orientations derived from 24 variants of the Kurdjumov-Sachs relationship as described

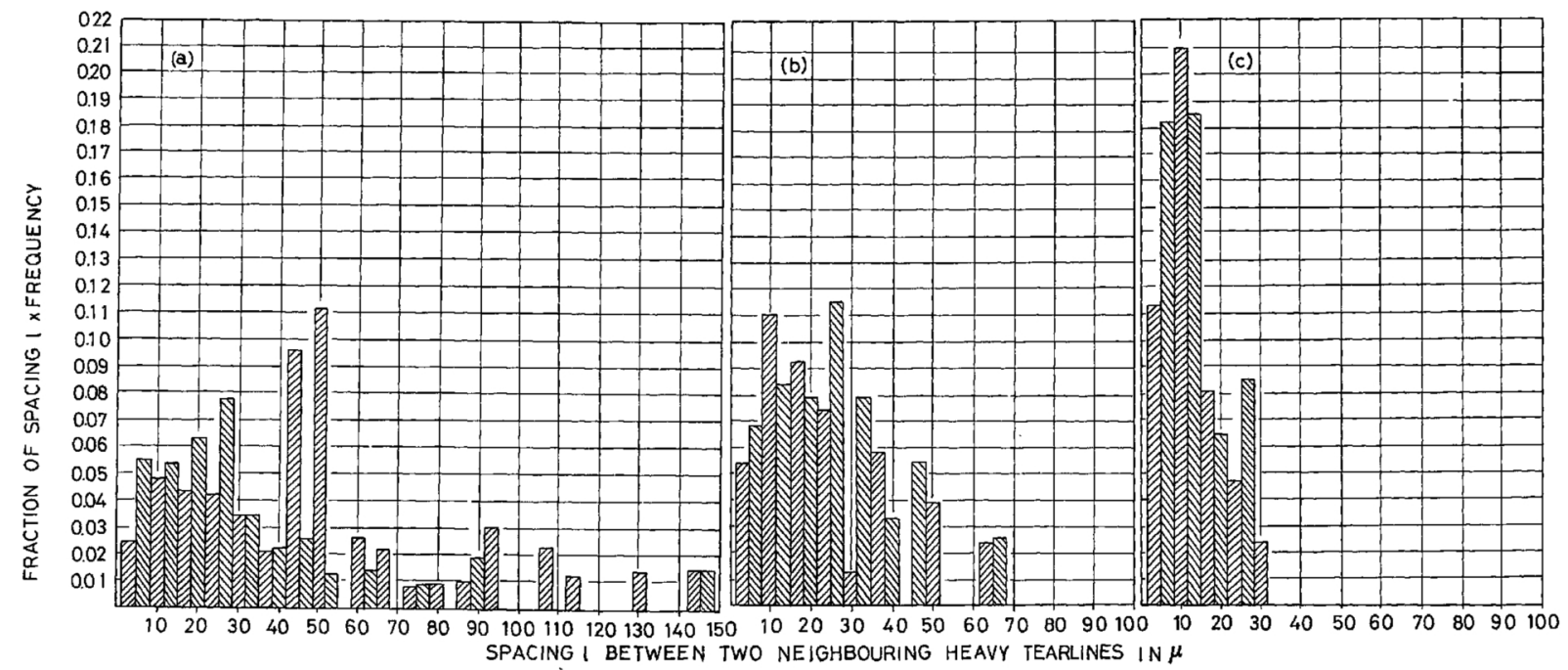

Fig. 1 Histogram obtained by lineal analysis of spacing between two heavy tear lines. The fraction of spacing is defined by $f_{j} \times l_{j} / \Sigma f_{i} \times l_{i}$, where $l_{i}$ is the spacing and $f_{i}$, the frequency of spacing $l_{i} \pm 1.25 \mu$

$\begin{array}{lllll}\text { (a) : Specimen A } & \text { (b) : Specimen B } & \text { (c) : Specimen C } & \dagger \text { Similar to kalling reagent. }\end{array}$ 
later and the black etched area corresponds to the other. The packet of martensite plates (or needles) which were formed by transformation following the same variant of the $\mathrm{K}-\mathrm{S}$ relationship is hereafter designated a 'co-variant packet'. In general a bundle is formed by alternation of two kinds of co-variant packets. There are two types of bundles :

Type 1: A bundle composed mainly of one kind of co-

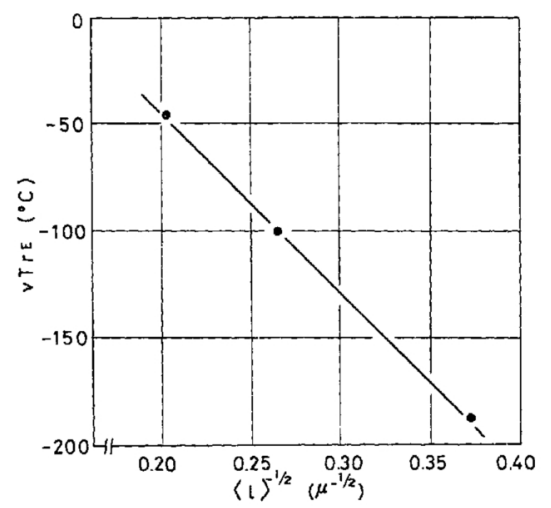

Fig. 2 Effect of the mean spacing $\langle b\rangle$ on transition temperature $v \operatorname{Tr}_{E}$

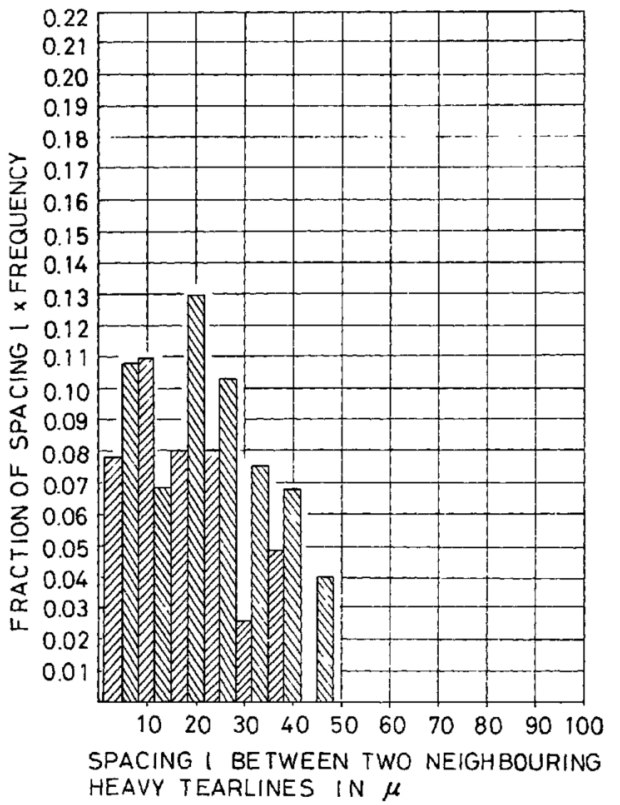

Fig. 3 Histogram obtained by lineal analysis of spacing between two heavy tear lines

Specimen : as iced brine quenched state from 1000 ${ }^{\circ} \mathrm{C}$ after 30 -min heating
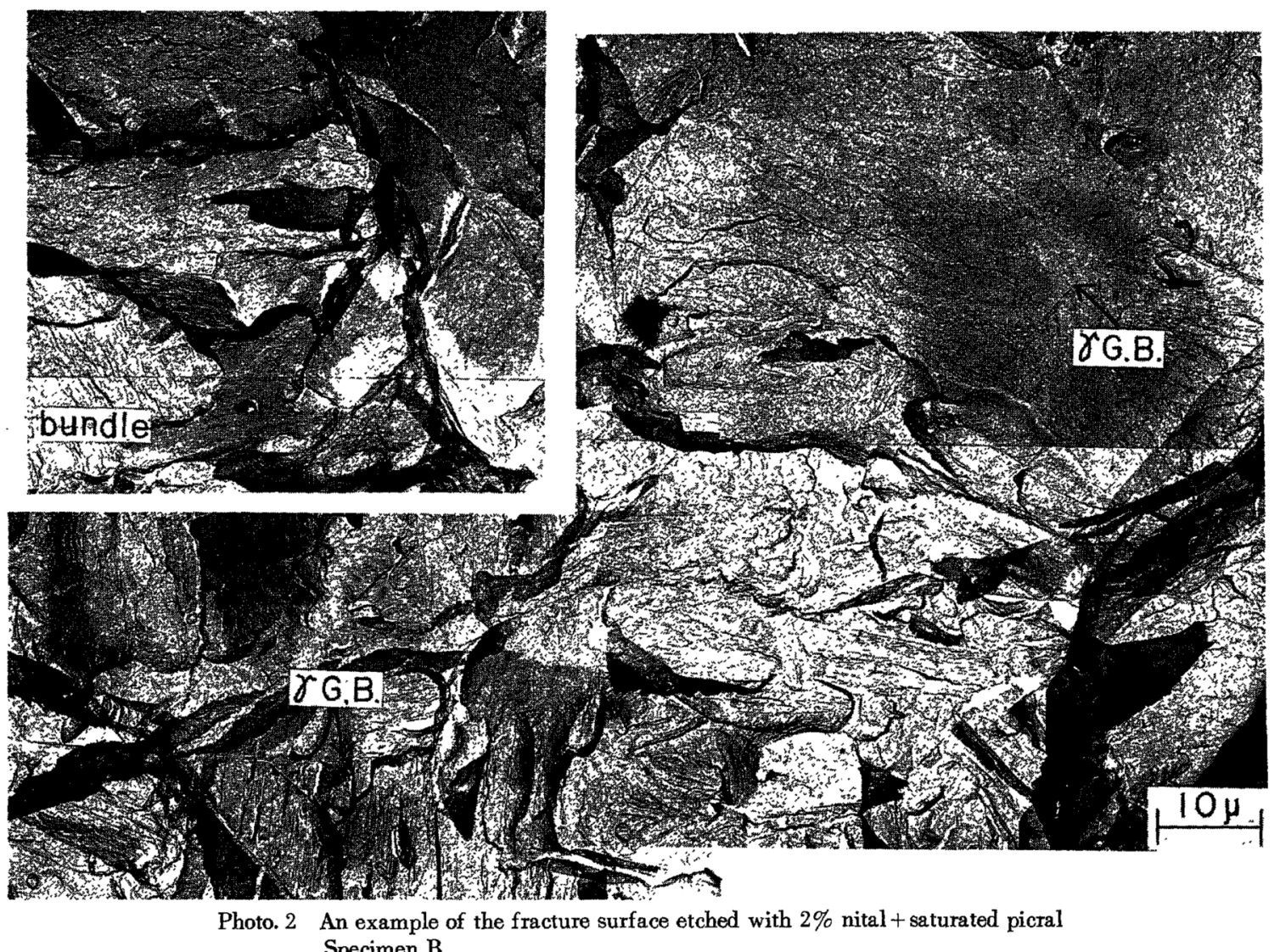

variant packet (A bundle etched mainly in white or mainly in black): A bundle consists mainly of many parallel martensite plates or laths which were formed by transformation following the same variant of the K-S relationship, although many tiny co-variant packets formed by transformation following the other variant are observed within it; i.e., many tiny black stripes are observed within a white bundle and vise versa, as seen in Photo. 3/a).

Type 2: A bundle composed of alternations of two fairly large co-variant packets (black and white stripes with nearly same widths): In this case there are also some very tiny co-variant packets in each one, as seen in Photo. 3 (b). These co-variant packets are found to correlate 
(a)
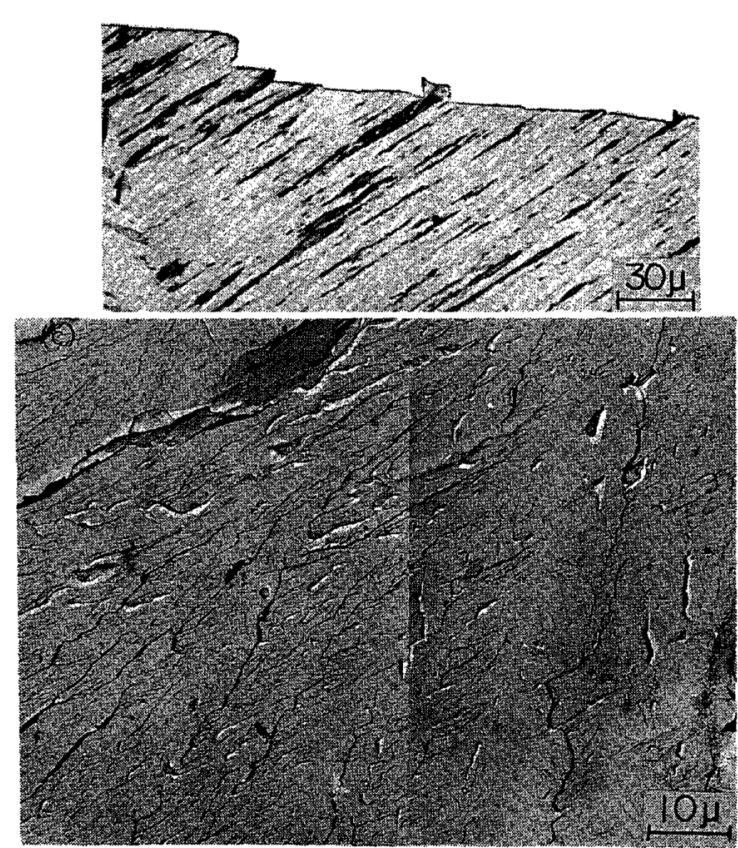

Photo. 3 Relation between fracture surface and microstructure

(a) (b) section of fracture surface

(c) (d) fracture surface

Specimen A
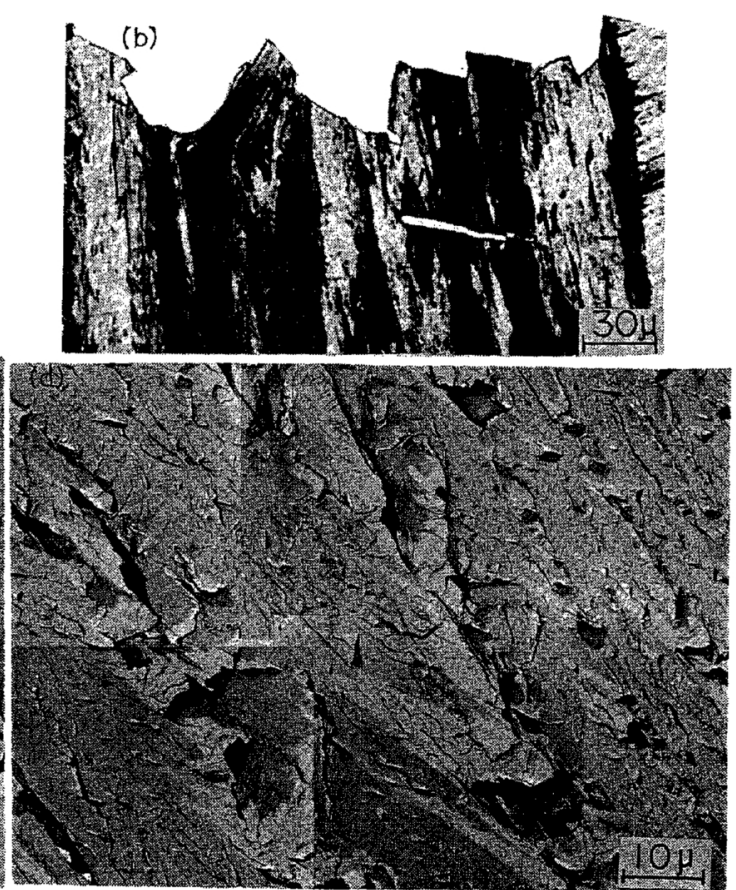

101
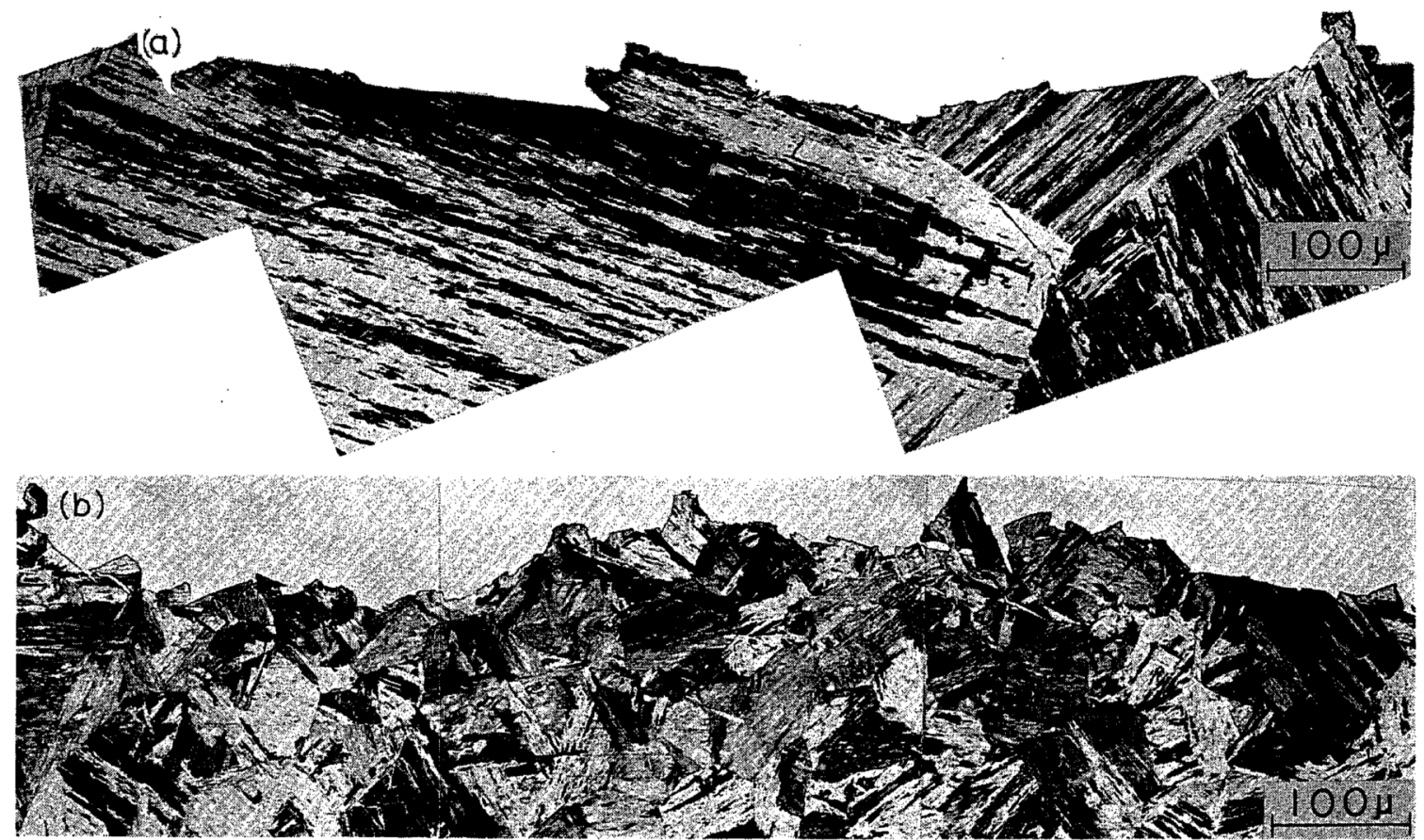

(c)

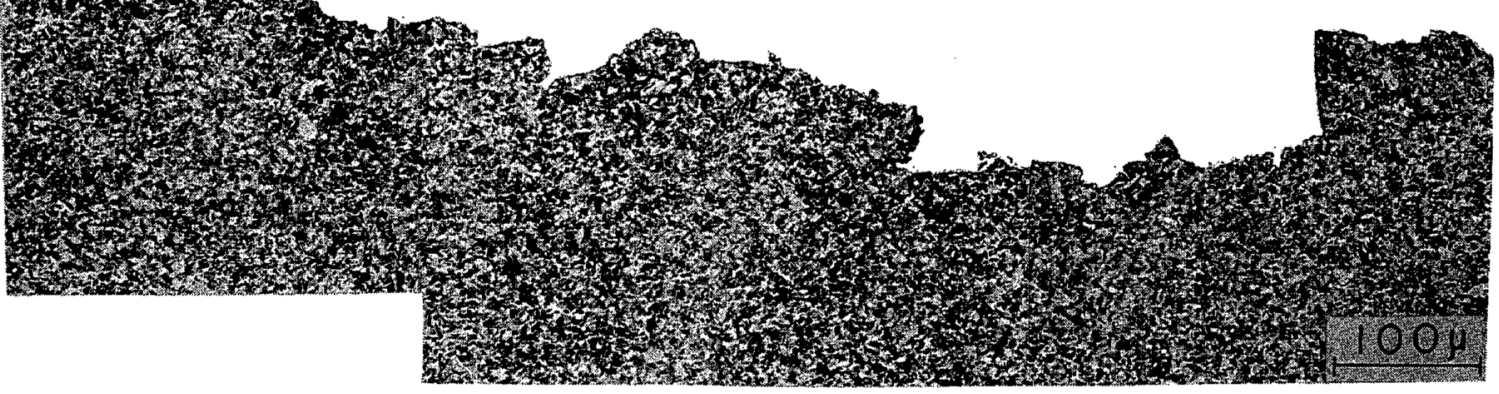

Photo. 4 Relation between fracture surface and microstructure

(a) : Specimen A (b) : Specimen B (c) : Specimen C 
with heavy tear lines as follows :

(1) In the bundle of type 1: The fracture surface is very flat throughout the bundle, although small tear lines are observed. It is to be noted that in the area where tear lines are observed within a bundle there exist minor co-variant packets (Photo. 3(a)).

(2) In the bundle of type 2 : The fracture surface usually changes its direction at the boundaries of the covariant packets, where heavy tear lines are observed. In some cases the fracture surfaces are parallel on both sides of a boundary, with tear lines on the boundary (Photo. 3 (b)).

Photos.3(c) and (d) show the replicas of the fracture surfaces; (c) correspond to the type 1 and (d) to the type 2 .

Photo. 4 shows the sections of the fracture surfaces of the specimens $A, B$ and $C$ with special reference to their microstructures. As the austenite grain size decreases, the size of the bundle and of the co-variant packet decreases and in all cases cleavage fracture facets correspond exactly to the co-variant packets.

\section{The crystallographic characteristics of the low carbon martensite}

It was found by selected area electron diffraction of the low carbon martensite that (1) a bundle consists of many martensite plates or laths formed by transformation followirg only two variants of the $\mathrm{K}-\mathrm{S}$ relationship. These two variants are frequently twin-related with each other. (2) The orientation of martensite plates or laths alters (according to their variant) by every several units, or more.

From analysis of the diffraction patterns which were taken successively from one end to the other of the bundle, it was found that there are two types of bundles. Photos. 5 and 6 show examples of the two types respectively. In Photo. 5 all the patterns consist of $(100)_{\alpha}$
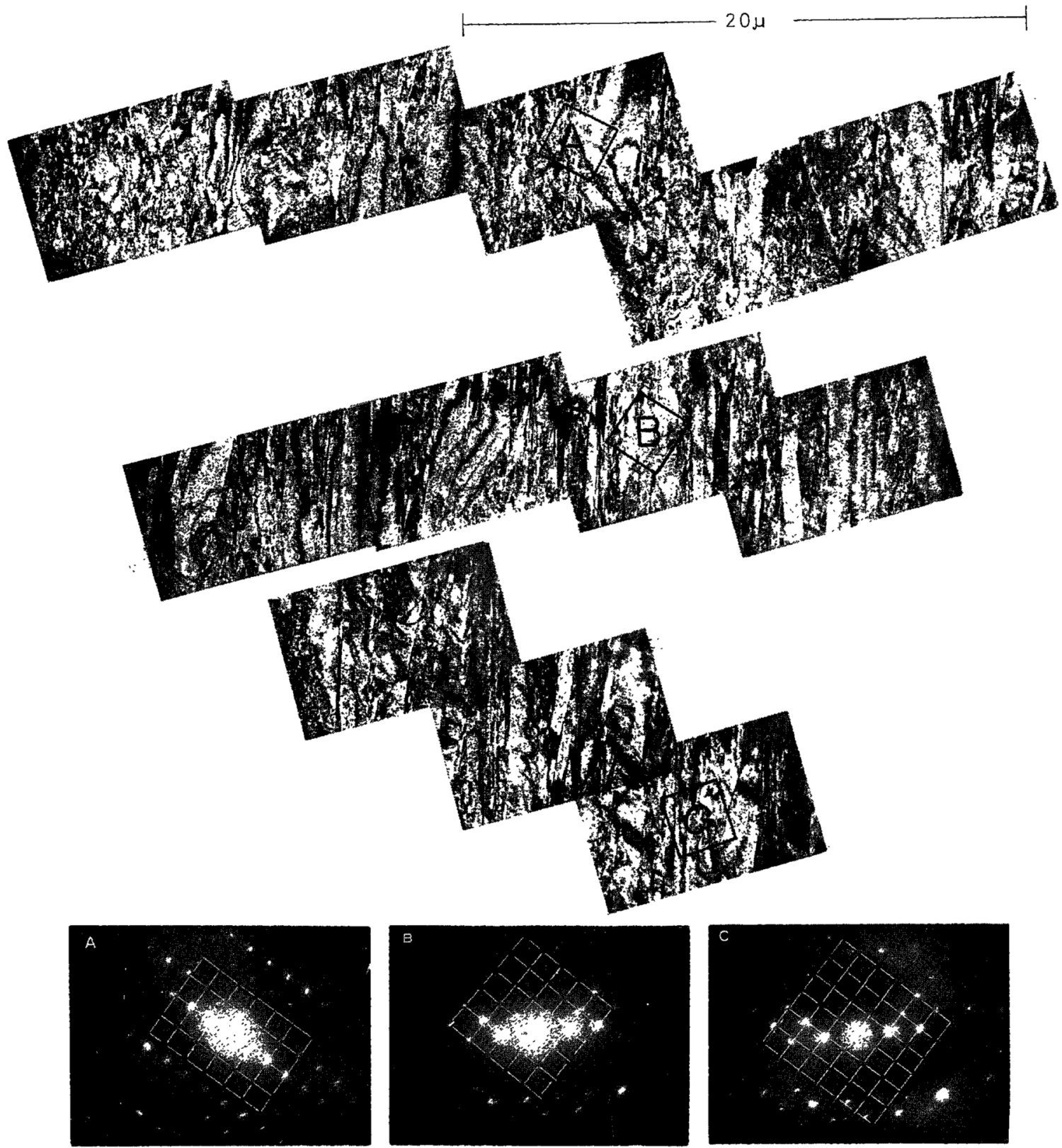

Photo. 5 Thin foil electron micrograph and selected area electron diffraction pattern of successive martensite plates or laths $\left((100)_{\alpha}\right.$ is very strong in every area) Specimen A 
and $(111)_{\alpha,}(100)_{\alpha}$ being very strong in the whole area extending over $100 \mu$. This shows that most plates or laths were formed by transformation following one variant of the K-S relationship, but there always exist a few plates or laths formed by transformation following the other variant. In Photo. 6 the bundle consists of the alternation of two kinds of area of a few micron width ; in one kind $(100)_{\alpha}$ is strong but $(111)_{\alpha}$ is weak and vice bundle boundary and boundary of co-variant packet extending through a bundle.

The sizes of microcracks were, for the most cases, from several tens to one hundred microns.

It should be noted that the following two types of heavy tear lines were observed within the surfaces of microcracks. (1) Heavy tear line corresponding to the small co-variant packet which does not extend through a bundle.

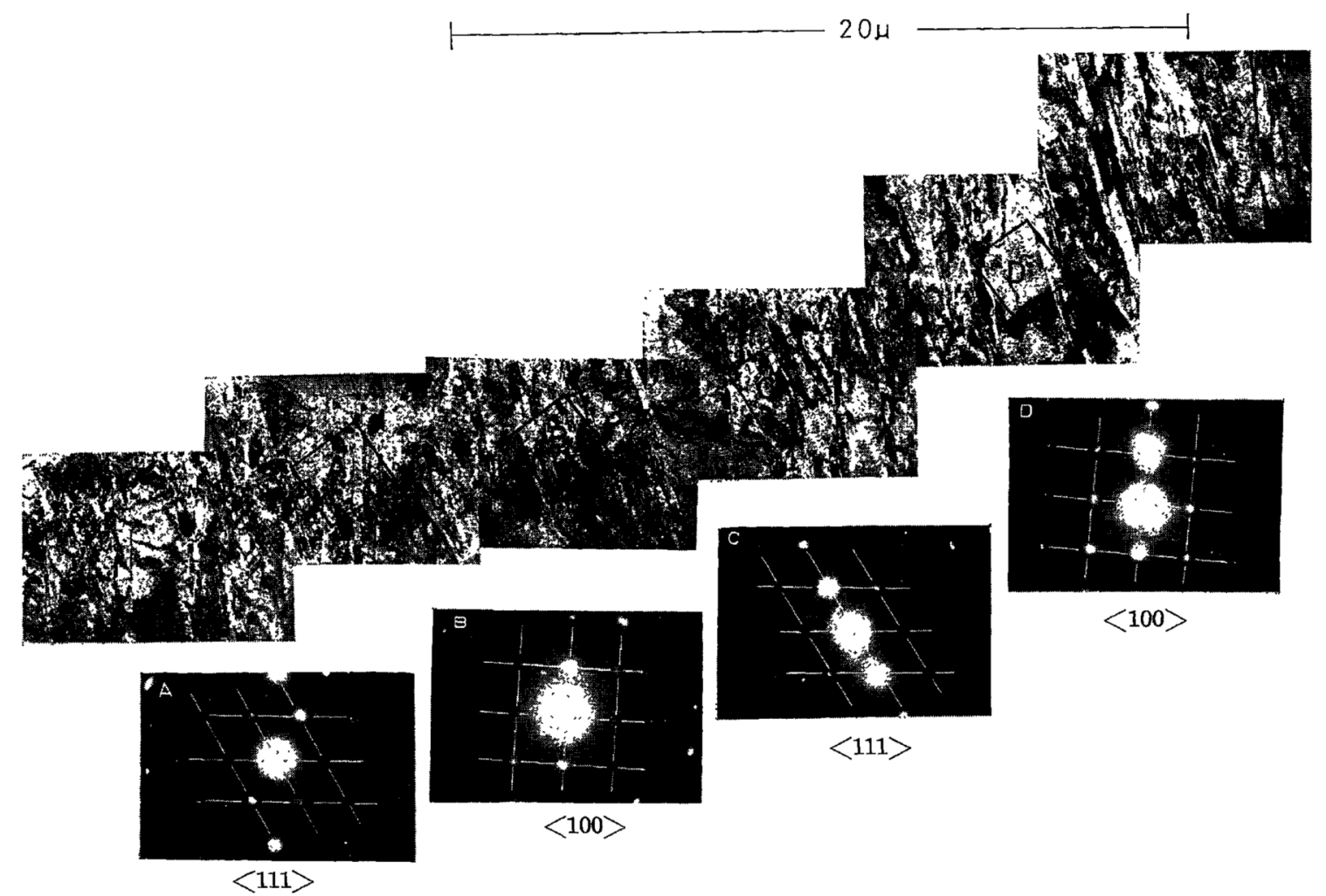

Photo. 6 Thin foil electron micrograph and selected area electron diffraction pattern of successive martensite plates or laths (alternation of the area where $(\mathbf{1 0 0})_{\alpha}$ is strong and the area where $(111)_{\alpha}$ is strong)

versa in the other kind.

These features explain the 'co-variant packet' defined in relation to the optical niicrograph, if it is redefined that the 'co-variant packet' is the packet of martensite plates or laths which were mostly formed by transformation following the same variants of the $\mathrm{K}-\mathrm{S}$ relationship.

Therefore, in a co-variant packet cleavage plane $\{100\}_{\alpha}$ is not continuous but quasi-continuous.

\section{Microcrack}

The observation of microcracks in the ductile temperature region furnishes a means of testing whether the above-mentioned heavy tear lines are the results of the effective barriers to the cleavage crack propagation ${ }^{(3)}$ and correspond to the effective grain boundaries. Cleavage microcracks (hereafter abbreviated 'microcrack') were found to exist on the fracture surfaces of the specimen tested immediately above the transition temperature, which appeared 100\% ductile on visual observation. Photo. 7 shows an example.

Heavy tear lines surrounding microcracks were analogous to those observed at the austenite grain boundary,

(3) G. T.Hahn et al. : Fracture, John Wiley and Sons, New York, (1959), p. 91.
(2) Heavy tear line of irregular shape formed by joining two parallel cleavage cracks on different crystal planes.

The following type of heavy tear line was not observed within the surfaces of microcracks but did not also constitute any microcrack boundary; heavy tear line subdividing the area surrounded by two nearly parallel heavy tear lines corresponding to a large co-variant packet.

Typical examples are shown in Photo. 8, together with their schematic drawings. A mechanism to produce such a fracture pattern will be discussed later.

\section{Discussion}

In the present work the cleavage fracture pattern was correlated with the microstructure and all the heavy tear lines were confirmed to be related with the crystallographic features of the low carbon martensite. The observation of microcracks suggests ; the interpretation in the previous paper ${ }^{(2)}$ that the spacing $l$ between neighbouring two heavy tear lines corresponds to the effective grain size is unsatisfactory for the following reasons. Firstly, the mean spacing $l$ is smaller than the size of microcracks, and, secondly, some sorts of heavy tear lines are observed within the surface of microcracks. It should 
be, therefore, necessary to eliminate the ineffective heavy tear lines. The heavy tear lines which are considered as ineffective are :
(1) Heavy tear lines corresponding to the small covariant packets. In this case the small packet is an island within the bundle. As judged from the direction of

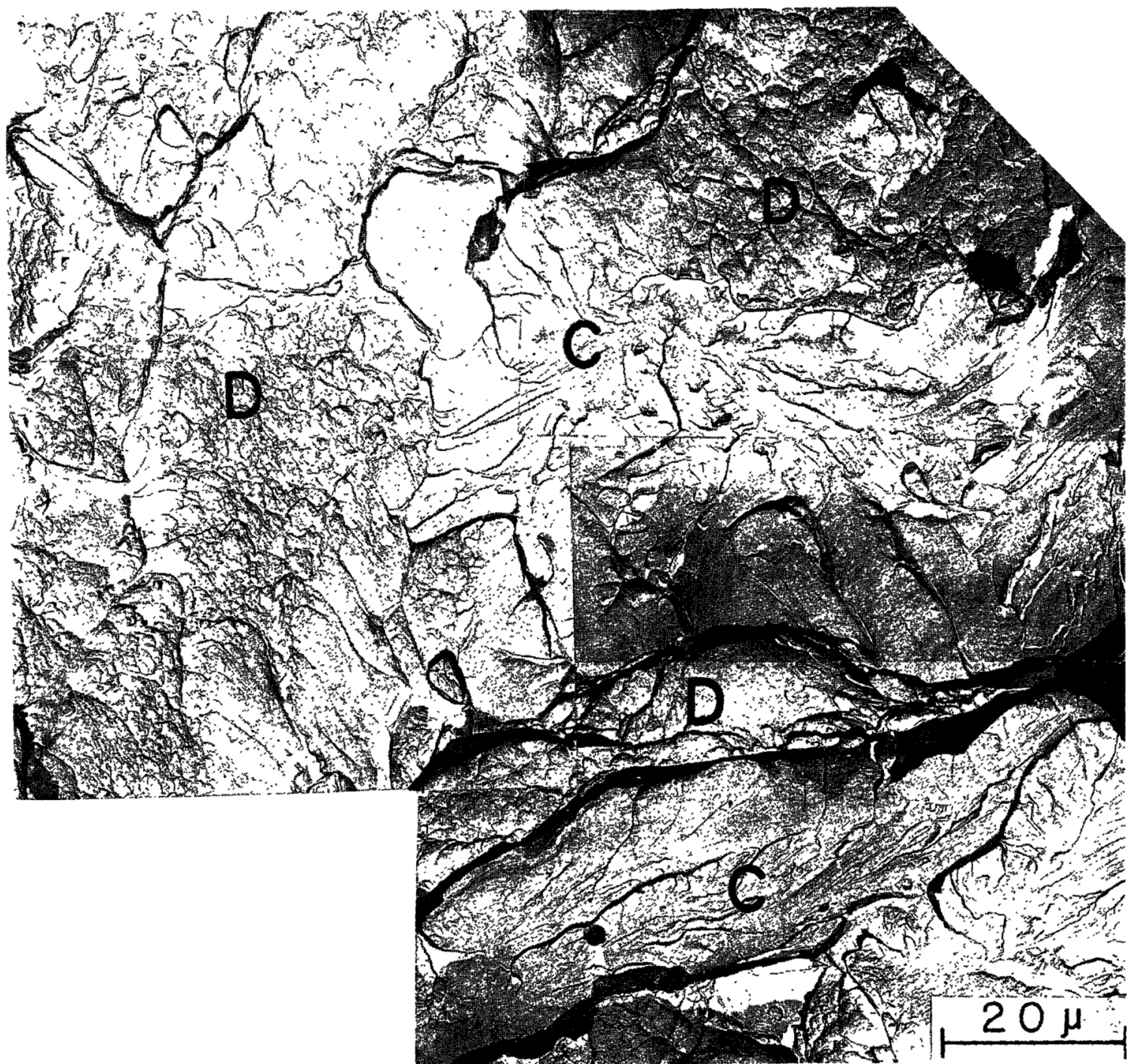

Photo. 7 An example of microcrack observed in the ductile temperature region

Testing temperature: $-40^{\circ} \mathrm{C}\left(v \operatorname{Tr}_{E}=-45^{\circ} \mathrm{C}\right) \quad$ Absorbed energy : $27.7 \mathrm{~kg}-\mathrm{m}$

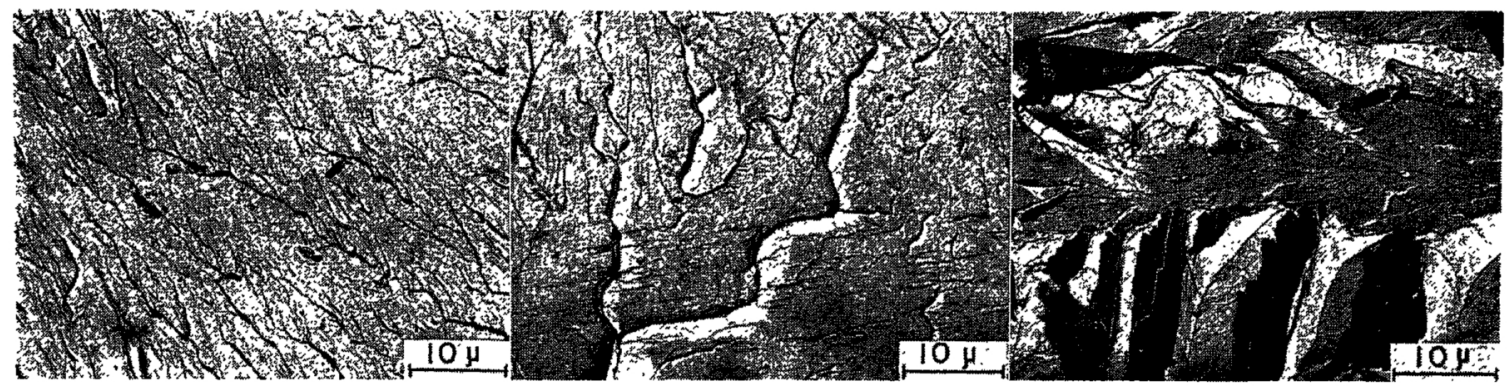

(1)

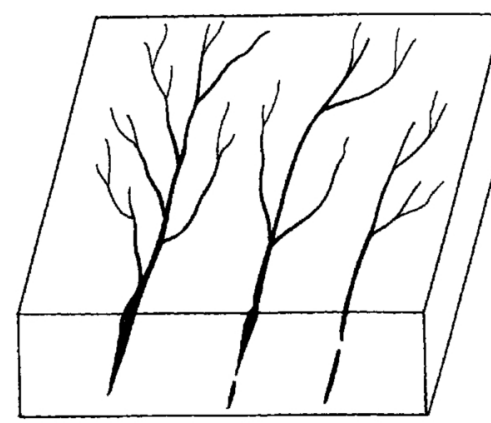

(2)

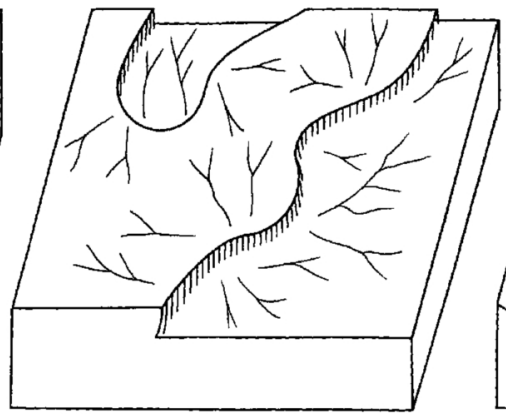

Photo. 8 Examples of ineffective tear lines
(3)

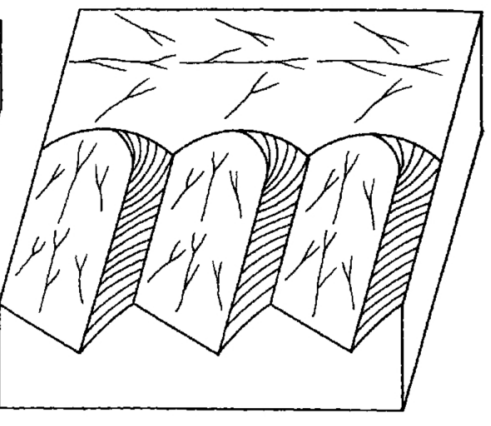




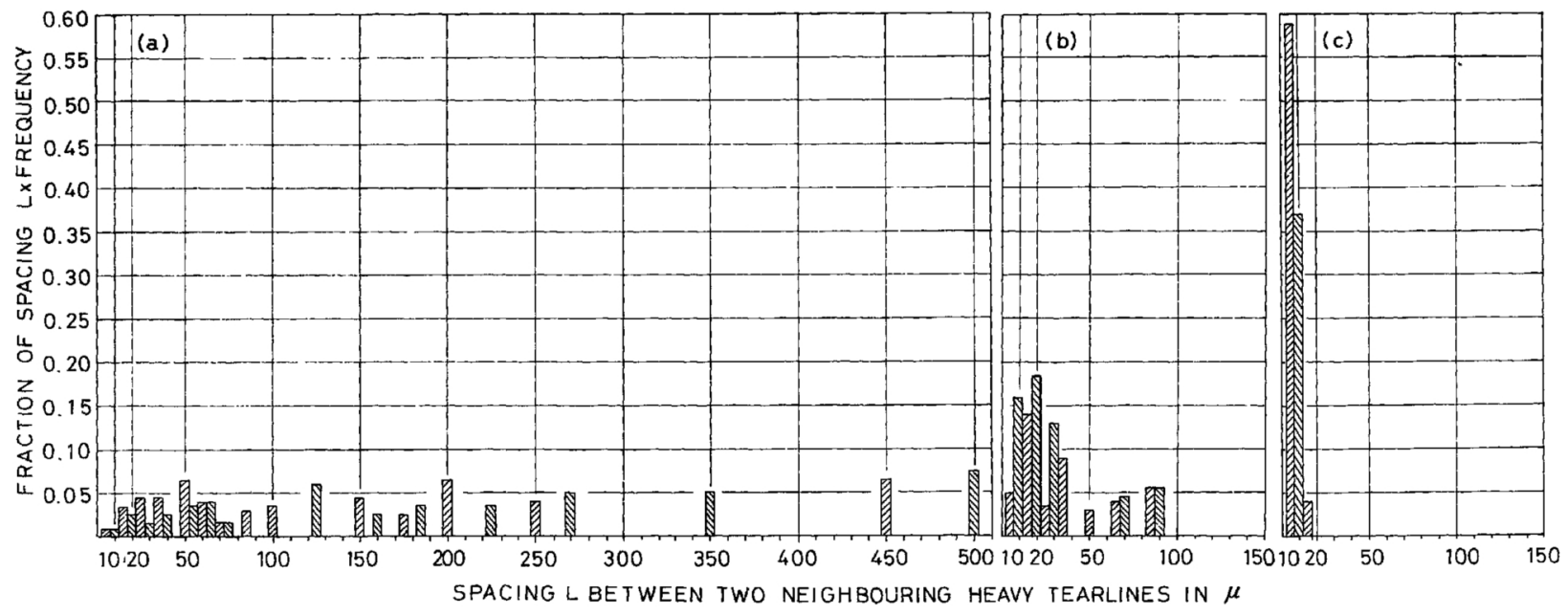

Fig. 4 Histogram obtained by lineal analysis of spacing $L$ between two effective tear lines $\begin{array}{lll}\text { (a): : Specimen A } & \text { (b) : Specimen B } & \text { (c) : Specimen C }\end{array}$

river pattern, it is supposed that the cleavage crack propagated in a bundle avoiding small packets and afterwords ductile tearing occurred in the region of small packets. Therefore, such tear lines are considered to be ineffective.

(2) Heavy tear line formed by joining two parallel cracks on different crystal planes. In this case tear lines are very irregular and supposed to be formed by chance. Therefore, it is evident that such a tear line is not effective.

(3) Heavy tear lines subdividing the area which is surrounded by two nearly parallel heavy tear lines. In this case the area surrounded by two nearly parallel heavy tear lines is supposed to correspond to the fairly large covariant packet. The mechanism producing such a pattern is considered as follows; as, the cleavage crack propagated from one co-variant packet to the neighbouring one it was divided into many parallel cracks on differing crystal planes required from continuity of fracture surface, and heavy tear lines were formed between subdivided cracks. Therefore, this type of tear line is considered to be ineffective.

Only heavy tear lines observed at austenite grain boundary, bundle boundary and boundary of co-variant packet extending through a bundle can be qualified as the effective grain boundary.

Spacings $L$ between effective tear lines mentioned above were measured, the result of which is illustrated in Fig. 4. Plotting $v \operatorname{Tr}_{E}$ against $\langle L\rangle^{-1 / 2}(\langle L\rangle$ is the statistical mean of $L$ ), a linear relationship can be obtained as shown in Fig. 5 .

It is concluded that the microstructure corresponding to the effective grain is the 'co-variant packet extending through a bundle'.

For the calculation of the effective surface energy of the cleavage crack it may be necessary to take into account the heavy tear lines rejected as ineffective. At first sight, the method of this analysis might seem to be somewhat arbitrary. However, it is a reasonable and perspicacious way to correspond the fracture of the martensitic steel to that of the ferritic steel. Therefore, the concept of the effective tear line is useful when it corresponds to the virtual ferrite grain boundary.

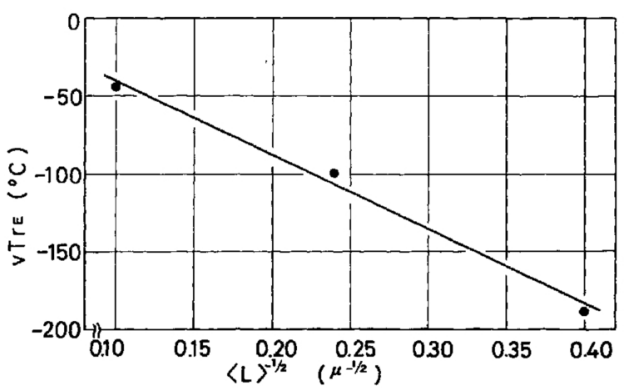

Fig. 5 Effect of the mean spacing $\langle L\rangle$ on transition temperature $v \operatorname{Tr}_{E}$

The fracture characteristic of low carbon martensite is different from that of medium carbon martensite ${ }^{(4)}$. This fact may be explained by the difference of the crystallographic. features between low carbon and medium carbon martensites ${ }^{(5)}$.

\section{Summary}

Main results on the relation between microstructure and fracture characteristics of low-carbon tempered martensite are summarized as follows :

(1) The microstructure corresponding to the effective grain is the "co-variant packet extending through a bundle".

(2) The reason why low carbon martensite fractures with large cleavage facets comes from the fact that almost all of martensite plates or laths in a packet have the same crystallographic orientation and the cleavage plane $\{100\}_{\alpha}$ is quasi-continuous throughout the packet.

\section{Aclnowledgments}

The authors wish to thank Prof. S. Mizushima, Dr. T. Ohtake for their interest in this research, Prof. Z. Nishiyama, and Dr. H. Mimura for their guidance and many helpful suggestions throughout this study, and Prof. J. Takamura for his helpful discussion.

(4) A. M. Turkalo: Trans. Met. Soc. AIME, 218 (1960), 24.

(5) to be published. 